\title{
Investigation of a Staphylococcus argenteus Strain Involved in a Chronic Prosthetic-Joint Infection
}

\author{
Alan Diot ${ }^{1}\left(\mathbb{D}\right.$, Virginie Dyon-Tafani ${ }^{1}$, Marine Bergot ${ }^{1}$, Jason Tasse ${ }^{2}(D$, \\ Patricia Martins-Simões ${ }^{1,3}$, Jérôme Josse ${ }^{1,4, * \mathbb{D}}$, Florent Valour ${ }^{1,4,5}$ and Frédéric Laurent $1,3,4,6$ \\ 1 CIRI-Centre International de Recherche en Infectiologie, Inserm, U1111, Université Claude Bernard Lyon 1, \\ CNRS, UMR5308, École Normale Supérieure de Lyon, Université Lyon, 69007 Lyon, France; \\ alandiot@hotmail.fr (A.D.); virginietafani@hotmail.fr (V.D.-T.); marine.bergot@univ-lyon1.fr (M.B.); \\ patricia.martins-simoes@chu-lyon.fr (P.M.-S.); florent.valour@chu-lyon.fr (F.V.); \\ frederic.laurent@univ-lyon1.fr (F.L.) \\ 2 BioFilm Control, 63360 Saint-Beauzire, France; jason.tasse@chu-lyon.fr \\ National Reference Center for Staphylococci, Hospices Civils de Lyon, 69004 Lyon, France \\ 4 Regional Reference Centre for Complex Bone and Joint Infection (CRIOAc Lyon), Hospices Civils de Lyon, \\ 69004 Lyon, France \\ 5 Department of Infectious Diseases, Hospices Civils de Lyon, 69004 Lyon, France \\ 6 Department of Bacteriology, Institute for Infectious Agents, Hospices Civils de Lyon, 69004 Lyon, France \\ * Correspondence: jerome.josse@univ-lyon1.fr
}

Received: 7 July 2020; Accepted: 28 August 2020; Published: 28 August 2020

\begin{abstract}
Staphylococcus argenteus is an emerging species responsible for infections comparable to those induced by Staphylococcus aureus. It has been involved in few chronic or persistent infections so far. In this study, we described a case of a persistent prosthetic-joint infection (PJI) affecting a young woman. We investigated in vitro the virulence traits of the incriminated S. argenteus strain (bone cell invasion, biofilm formation and induction of inflammation) and analyzed its genome, in comparison with two other strains of $S$. argenteus and two S. aureus isolates. It appeared that this S. argenteus PJI strain combined biofilm formation, osteoblast invasion and intracellular persistence abilities together with genes potentially involved in the escape of the host immune defenses, which might explain the chronicization of the infection.
\end{abstract}

Keywords: Staphylococcus argenteus; bone and joint infection; biofilm; intracellular; osteoblast

\section{Introduction}

Staphylococcus argenteus is a novel and emerging species belonging to the Staphylococcus aureus complex and, as such, was historically misidentified as $S$. aureus clonal complex 75 [1]. It causes infections comparable to those triggered by S. aureus, e.g., skin and soft tissue infections, bacteremia and sepsis, and therefore can be life-threatening. Sporadic cases have been reported mainly in Asia, Oceania and Pacific islands [2,3] and more rarely in Europe [4,5].

S. aureus virulence relies on a large panel of toxins such as the Panton-Valentine leucocidin (PVL) or phenol soluble modulins (PSMs) as well as adhesion factors such as microbial surface components recognizing adhesive matrix molecules (MSCRAMMs) or various enzymes including proteases [6]. In addition, $S$. aureus infections can become chronic and last over months due to three major bacterial strategies: biofilm formation, non-professional phagocytic cell (NPPC) invasion [7-10] and switching to a quasi-dormant and slow-growing phenotype called "small colony variant" (SCV) [11]. These three virulence factors allow bacteria to escape both the immune system and the action of most antimicrobials. Internalization into NPPCs involves staphylococcal fibronectin binding proteins (FnBPs) that link fibronectin from the extracellular matrix acting as a bridge to the cellular $\beta 1$ integrin [12-14]. Once into 
the cells, S. aureus can survive using the cell as a shelter to hide from extracellular antibiotics and the host immune system. In the same way, bacterial organization in biofilms offers a physical barrier against the immune system and antimicrobials [15]. In addition, it is now rather accepted that the reduced metabolism and growth rate associated with biofilm lifestyle, as well as changes in gene expression of biofilm-embedded bacteria, also provide a protection from both antibiotics and the immune system [16-19]. Factors and mechanisms responsible for S. aureus virulence have been extensively investigated but only few studies and data are available for S. argenteus. Genes encoding the PVL and staphylococcal enterotoxins have been identified [20-22], and a switch to a SCV phenotype during a chronic infection or in presence of high concentrations of amikacin has also been reported [23]. However, biofilm formation and cell invasion have not been extensively investigated yet in S. argenteus.

In the present study, we identified a strain of $S$. argenteus responsible for a chronic prosthesis-joint infection (PJI) in a young woman and assessed bacterial factors possibly responsible for the chronicization. After describing the clinical case, we investigated its in vitro phenotypical traits, i.e. cell invasion, biofilm production, cytotoxicity, induction of cytokines secretion as well as its genotypical traits. Results were compared with those obtained with two other strains of S. argenteus, as well as two $S$. aureus strains used as references.

\section{Results}

\subsection{Clinical History}

In 2006, a 20-year-old Cambodian woman benefited from a total hip arthroplasty with shortening subtrochanteric osteotomy for a left hip osteoarthrosis secondary to a developmental dysplasia. A hip prosthesis and osteosynthesis devices (plates and screws) were implanted in the femur. Postoperative course was marked by a bacteremia originating from a peripheral catheter-related phlebitis due to a bacterium identified at this time as a methicillin-susceptible Staphylococcus aureus (MSSA). The patient was treated by a two-week course of intravenous cloxacillin (Figure 1A). Six months later, she developed chronic mechanical pain of the left hip. In February 2008, after more than a year of mechanical pain, the ablation of osteosynthesis devices was performed with prosthesis retention (Figure 1A,B).

Systematic periprosthetic bone tissue samples yielded few colonies of a bacterium identified as MSSA. A combination of pristinamycin and rifampin was prescribed for three months. As pain worsened, a two-stage exchange strategy was planned in October 2008 (Figure 1A,C). Bone tissue sampled before the surgery and during the prosthesis ablation were still positive for MSSA (Figure 1A). The postoperative treatment using intravenous cloxacillin and oral ofloxacin was continued for six weeks, and then switched to an oral combination of ofloxacin and fusidic acid. A prosthesis reimplantation was performed after three months of treatment (Figure 1A,D). Bacteriological samples remained sterile at that time. Antimicrobial therapy was stopped three months later, and, after a five-year follow-up, the patient was doing well, with no clinical or radiological symptoms of recurrence.

The strain isolated during the last episode of recurrence was originally identified as a methicillin-sensitive Staphylococcus aureus using Vitek2 (Biomérieux) for identification and antibiotic susceptibility determination. The sequence type of this strain was defined as ST75, using DNA microarrays and identibac S. aureus Genotyping (Alere) [24,25]. However, when its genome was sequenced for the need of a recent research project, we acknowledged this strain as belonging to the Staphylococcus argenteus species. Since all the strains identified during this case were MSSA harboring the same antimicrobial susceptibility profile (Table 1), we wonder if all the strains could actually be S. argenteus misidentified as MSSA. However, we cannot clarify this point as we cannot sequence the isolates from the previous episodes of infection because the samples are no longer available.

This case would thus be one of the few chronic S. argenteus infections described and we decided to investigate the cell invasion and the related cellular response, as well as the biofilm production properties of this strain $\left(\right.$ S. argenteus $^{\mathrm{PJI}}$ ) using in vitro tests. 

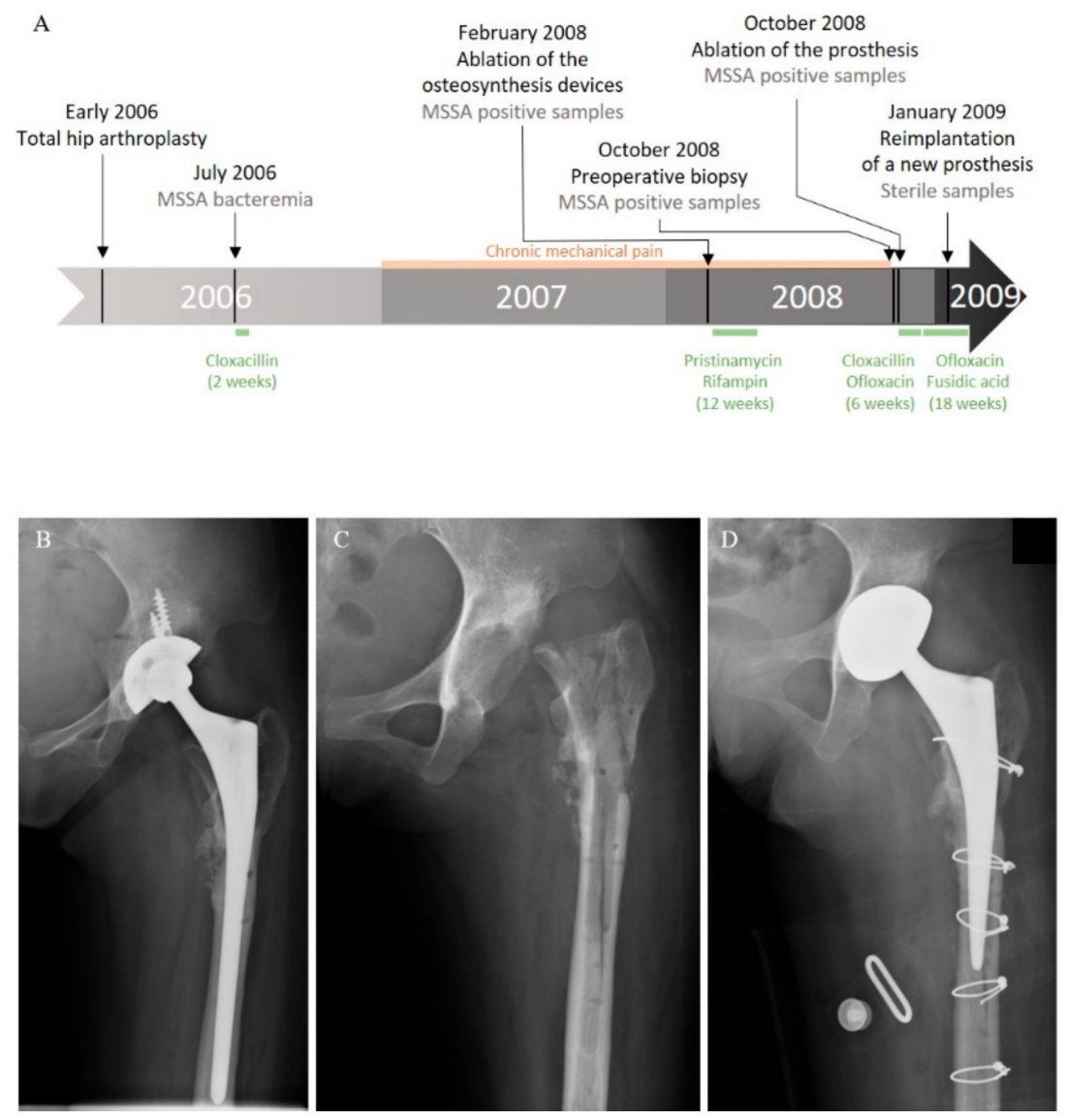

Figure 1. Clinical history of the case. (A) Schematic representation of the course of the infection and treatments. Left hip X-ray: (B) after osteosynthesis device ablation; (C) after prosthesis removal; and (D) after reimplantation.

Table 1. Antibiogram of the S. argenteus ${ }^{P J I}$ strain. $\mathrm{R}$ is for resistant and $\mathrm{S}$ is for sensitive according to the CASFM guidelines.

\begin{tabular}{cccccc}
\hline Antibiotic & Susceptibility & Antibiotic & Susceptibility & Antibiotic & Susceptibility \\
\hline Peni-G & R & Erythromycin & R & Vancomycin & S \\
\hline Peni-M & S & Lincomycin & R & Teicoplanin & S \\
\hline Gentamicin & S & Synergistins & S & Fosfomycin & S \\
\hline Tobramycin & S & Rifampicin & S & Fusidic acid & S \\
\hline Kanamycin & S & Nitrofurantoin & S & Linezolid & S \\
\hline Tetracyclin & S & Cotrimoxazol & S & & \\
\hline Minocyclin & S & Ofloxacin & S & & \\
\hline
\end{tabular}

\subsection{Cellular Invasion Properties}

We first assessed the capacity of $S$. argenteus $^{\mathrm{PJI}}$ to adhere and invade non-professional phagocytic cells, osteoblastic MG63 cells. Our results show an ability for cellular adhesion that appears similar to that of the poorly invasive $S$. aureus $8325-4$ (Figure $2 \mathrm{~A}, 2.44 \pm 1.84$ vs. $1.69 \pm 1.23 \times 10^{6} \mathrm{CFU}$ ) and slightly weaker than the one of the highly invasive $S$. aureus 29213 (Figure 2A, $2.44 \pm 1.84$ vs. $7.80 \pm$ $\left.4.82 \times 10^{6} \mathrm{CFU}\right)$. 
A

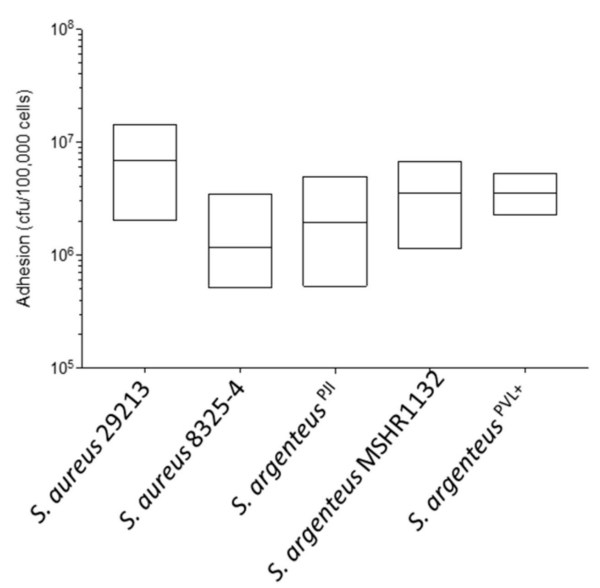

B

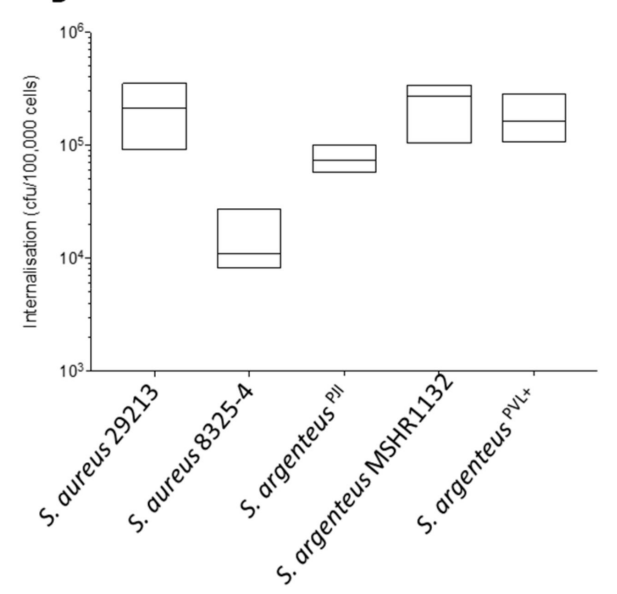

Figure 2. Properties of adhesion and internalization of the S. argenteus ${ }^{\mathrm{PII}}$ strain. (A) The adhesion to MG63 cells after $2 \mathrm{~h}$ of contact of the S. aureus and S. argenteus strains is expressed in CFU/100,000 cells. S. argenteus $^{\mathrm{PJI}}$ adhere to the cells with a similar efficacy than all the strains tested. (B) The intracellular inoculum recovered after $2 \mathrm{~h}$ of contact and $1 \mathrm{~h}$ of gentamycin treatment to kill the extracellular bacteria is expressed in CFU/100,000 cells. The S. argenteus ${ }^{\mathrm{PJI}}$ responsible for the chronic PJI showed an intermediate internalization between the poorly invasive $S$. aureus $8325-4$ and the invasive $S$. aureus 29213 or S. argenteus MSHR1132, S. argenteus ${ }^{\mathrm{PVL}+}$ strains.

When compared to the other $S$. argenteus strains, the cellular adhesion was also in the same range (Figure 2A, $2.44 \pm 1.84$ vs. $3.79 \pm 2.21$ and $3.65 \pm 1.12 \times 10^{6} \mathrm{CFU}$ for S. argenteus MSHR1132 and S. argenteus $^{P V L+}$ respectively).

At $3 \mathrm{~h}$ pi, S. argenteus $^{\mathrm{PJI}}$ was internalized more efficiently into MG63 cells than S. aureus 8325-4 (Figure $2 \mathrm{~B}, 7.47 \pm 1.46$ vs. $1.25 \pm 0.59 \times 10^{4} \mathrm{CFU}$ for S. argenteus ${ }^{\mathrm{PJI}}$ vs. S. aureus $\left.8325-4\right)$. However, intracellular bacterial load appeared lower than those of $S$. aureus 29213 (Figure 2B, $7.47 \pm 1.46 \mathrm{vs}$. $20.78 \pm 9.04 \times 10^{4} \mathrm{CFU}$ ) or of the other $S$. argenteus strains that also proved effective at invading cells (Figure $2 \mathrm{~B}, 7.47 \pm 1.46$ vs. $23.37 \pm 8.98$ and $15.78 \pm 5.35 \times 10^{4} \mathrm{CFU}$ ).

In osteoblastic cells, $S$. aureus is known to mainly use the $\beta 1$-integrin as a receptor to trigger its internalization. In our study, treatment with blocking anti- $\beta 1$ integrin antibodies resulted in a reduction of $S$. argenteus internalization by more than $95 \%$, with a mean of $3.1 \%$ of internalization capacity left after treatment (Figure 3), which was comparable to S. aureus 8325-4 (Figure 3, 3.4\% of internalization capacity left), suggesting that $S$. argenteus internalization pathway uses the same receptor or trigger.

Two other $S$. argenteus isolates tested showed similar results after anti- $\beta 1$ integrin treatment with a mean of $4.7 \%$ of internalization capacity left after treatment (Figure 3).

Overall, these results demonstrate that all tested $S$. argenteus strains were able to invade osteoblasts using a $\beta 1$ integrin-dependent mechanism.

\subsection{Intracellular Survival}

Interestingly, $24 \mathrm{~h}$ after internalization, the intracellular bacterial charge of the clinical S. argenteus isolate stayed similar to the one observed at $3 \mathrm{~h}$ post-infection (pi) (Figure 4A, 6.75 vs. $7.47 \times 10^{4} \mathrm{CFU}$ at 24 and $3 \mathrm{~h}$ pi, respectively).

After three days pi, the intracellular inoculum decreased by one log, reaching $6.07 \times 10^{3} \mathrm{CFU}$. This bacterial load tended to be higher than those of the two $S$. aureus strains at the same timepoint (Figure 4A, 2.08 and $1.53 \times 10^{3}$ CFU/100000 cells for 29213 and 8325-4 respectively). Focusing on S. argenteus strains, $S$. argenteus ${ }^{\mathrm{PJI}}$ behaved similarly to S. argenteus MSHR1132, although the latter displayed higher intracellular inoculum after 24 and $72 \mathrm{~h}$ (Figure $4 \mathrm{~A}, 1.64 \times 10^{5}$ and $1.38 \times 10^{4} \mathrm{CFU}$ for MSHR1132 at 24 and $72 \mathrm{~h}$ ). The S. argenteus ${ }^{\mathrm{PVL}+}$, on the other hand, was not able to maintain its intracellular inoculum between 3 and $24 \mathrm{~h}$ pi $\left(1.58 \times 10^{5}\right.$ and $4.36 \times 10^{4} \mathrm{CFU}$ at 3 and $24 \mathrm{~h}$ pi). 


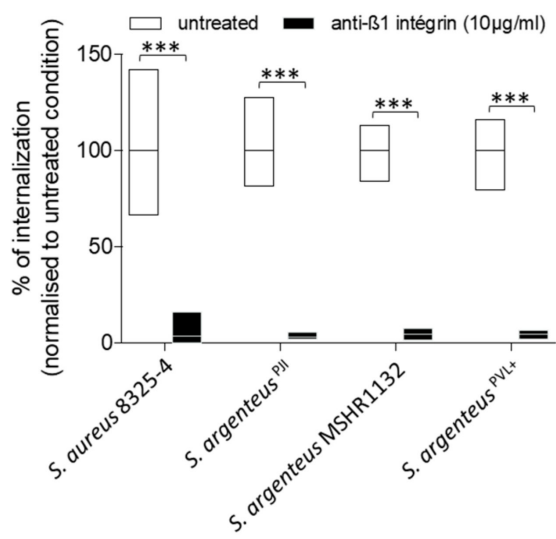

Figure 3. The internalization of $S$. argenteus strains into MG- 63 cells requires $\beta 1$ integrin. A pre-treatment of MG63 cells with a blocking anti- $\beta 1$ integrin antibody inhibits more than $90 \%$ of $S$. aureus and $S$. argenteus strains internalization. Mann-Whitney test was performed for statistical analysis. ${ }^{* * *}$ means $p<0.001$.
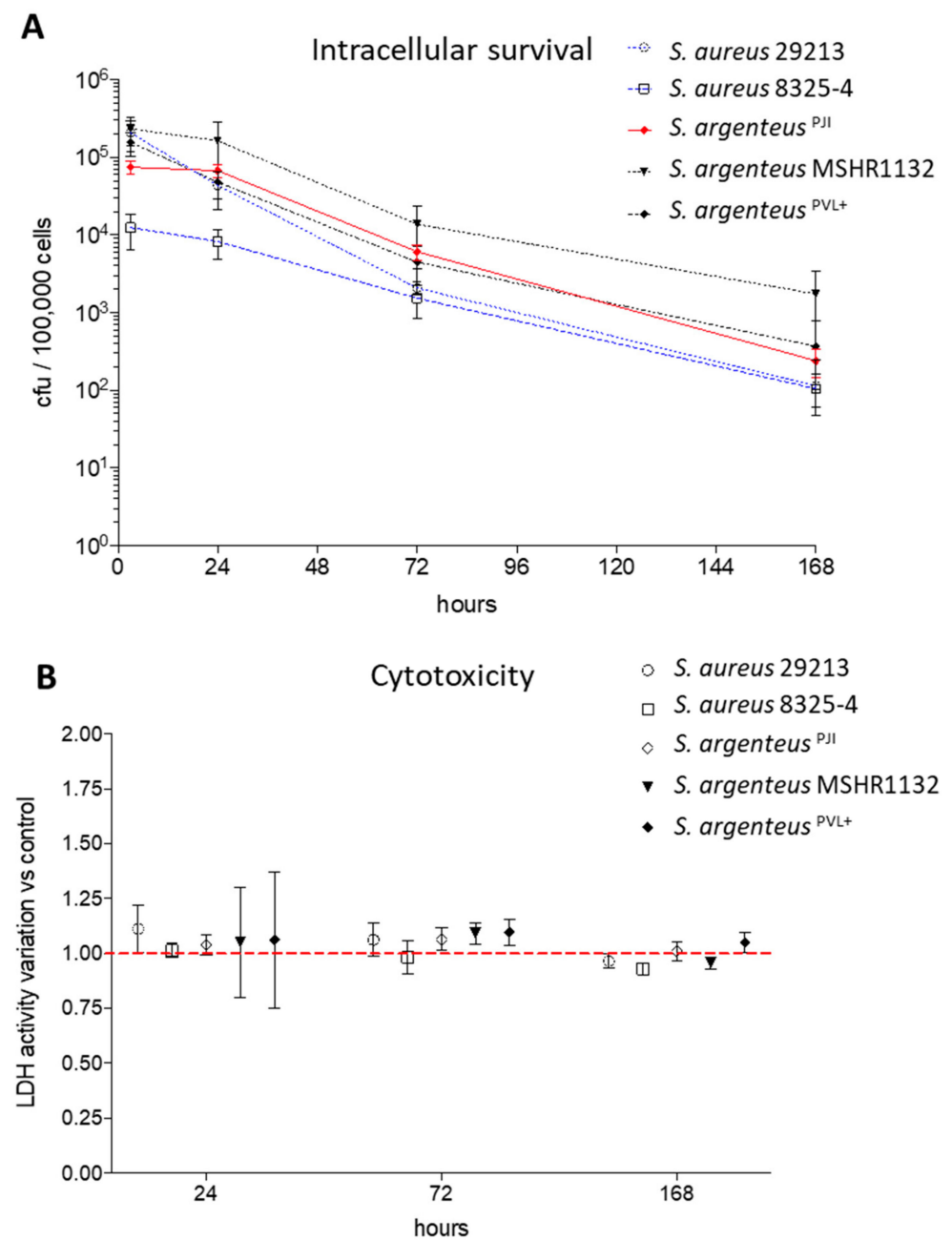

Figure 4. Intracellular survival and cytotoxicity induced by the S. argenteus ${ }^{\mathrm{PJI}}$. (A) The intracellular inoculum of S. aureus $(29213,8325-4)$ and S. argenteus strains (S. argenteus ${ }^{\mathrm{PJI}}$, S. argenteus MSHR1132, S. argenteus $^{P V L+}$ ) was followed over seven days, expressed in CFU/100,000 cells, and showed a better persistence for the S. argenteus strains. (B) The cytotoxicity induced by the strains has been assessed by following the LDH release in the extracellular medium. The data are presented as variation when compared to the relative non-infected control for each tested time of infection No cytotoxicity is observed for any strain. 
After seven days, a decrease of approximately 1-Log was observed when compared to intracellular inoculums at $72 \mathrm{~h}$ pi for all the strains (Figure $4 \mathrm{~A}$ ). However, intracellular bacteria were still detectable in all the conditions.

Altogether, these results point out that the S. argenteus isolates can survive intracellularly, as previously observed for $S$. aureus. However, the intensity of intracellular survival varies between the different $S$. argenteus strains. In our in vitro model, this could result either from a better intracellular life adaptation or from a decreased cytotoxic power that would limit the release of bacteria in the lethal extracellular environment that contains gentamicin.

\subsection{Cell Death Induction by Intracellular Staphylococci}

We evaluated the cytotoxic effect induced by intracellular bacteria by measuring the release of $\mathrm{LDH}$ at $24 \mathrm{~h}, 72 \mathrm{~h}$ and seven days after infection. Overall, we observed that the strains used in this study displayed a poor cytotoxic effect on non-professional phagocytic cells such as MG63, whether they are S. aureus or S. argenteus strains, expressing or not the PVL. After $24 \mathrm{~h}$ of infection, the cell death induced was still similar to what was observed for the invasive strain 29213 (Figure 4B, $104 \pm 4 \%$ vs. $111 \pm 11 \%, 101 \pm 3 \%, 105 \pm 25 \%$ and $106 \pm 31 \%$ for S. argenteus ${ }^{\mathrm{PJI}}$ vs. S. aureus 29213 , S. aureus $8325-4$, S. argenteus MSHR1132 and S. argenteus ${ }^{P V L+}$, expressed relatively to non-infected cells). The results were all in the same range at $72 \mathrm{~h}$ and seven days after infection (Figure $4 \mathrm{~B}$ ).

These results suggest that the better intracellular survival of S. argenteus ${ }^{\mathrm{PJI}}$ is likely due to a better adaptation to the intracellular compartment.

\subsection{Induction of a Cytokinic Response}

We investigated the release of interleukin 6 (IL-6), a key cytokine in inflammation regulation, and granulocyte macrophage colony stimulating factor (GM-CSF), a stimulating factor for neutrophils and macrophages, by infected osteoblasts, once the infection was established and the bacteria were persistent in the in vitro set up, i.e., $72 \mathrm{~h}$ after infection.

First, we observed that S. argenteus MSHR1132 and S. argenteus ${ }^{\mathrm{PVL}+}$ were among the strongest inducers of inflammation, with levels of secreted IL-6 similar or higher than the ones induced by the invasive S. aureus 29213 (Figure 5A, 21.3 and 23.6 vs. $17.2 \mathrm{pg} / \mathrm{mL}$ for S. argenteus MSHR1132 and S. argenteus $^{P V L+}$ vs. 29213).

Conversely, S. argenteus ${ }^{\mathrm{PJI}}$ appeared as a weaker inducer of inflammation with a concentration of secreted IL-6 similar to the poorly invasive S. aureus 8325-4 (Figure 5A, 6.5 vs. 5 vs. $4.8 \mathrm{pg} / \mathrm{mL}$ for S. argenteus ${ }^{\mathrm{PJI}}$ vs. S. aureus $8325-4$ vs. non-infected cells).

In line with this weak induction of inflammation, S. argenteus ${ }^{\mathrm{PII}}$ induced a release of GM-CSF similar to S. aureus 8325-4 and closer to the levels observed for the non-infected cells (Figure 5B, 29.1 vs. 25.6 vs. $18.6 \mathrm{pg} / \mathrm{mL}$ for $S$. argenteus vs. $8325-4$ vs. non-infected cells). GM-CSF release by osteoblasts infected by S. aureus 29213 or the two other S. argenteus was at least 2.5 -fold higher (71.7 vs. 73.2 vs. $69 \mathrm{pg} / \mathrm{mL}$ for S. aureus 29213 vs. S. argenteus MSHR1132 vs. S. argenteus $^{P V L+}$ ).

Together, these results suggest that $S$. argenteus ${ }^{\mathrm{PII}}$ induced a weak inflammatory response compared to the two other S. argenteus strains, which seems logical in a context of chronic and persistent PJI.

\subsection{Assessment of the Ability to Form Biofilm}

The other bacterial strategy used to shelter from the extracellular environment and antibiotics and persist is to form biofilm. First, we assessed the early biofilm formation capacity using the BioFilm Ring Test ${ }^{\circledR}$ [26]. The results highlight no difference between strains, classifying the five tested strains as moderate early biofilm producers (Figure 5).

We then investigated the formation of mature biofilm (after $24 \mathrm{~h}$ ) in absence or presence of glucose, using the crystal violet staining method. Indeed, addition of glucose in the culture medium promotes the formation of biofilm by S. aureus, especially MRSA strains [27]. In absence of glucose, S. argenteus ${ }^{\mathrm{PJI}}$ 
was able to produce a mature biofilm in a way similar to the two $S$. aureus strains (Figure 6, $\mathrm{OD}_{590}=0.23$ vs. 0.6 vs. 0.26 for S. argenteus vs. S. aureus $8325-4$ vs. S. aureus 29213$)$.
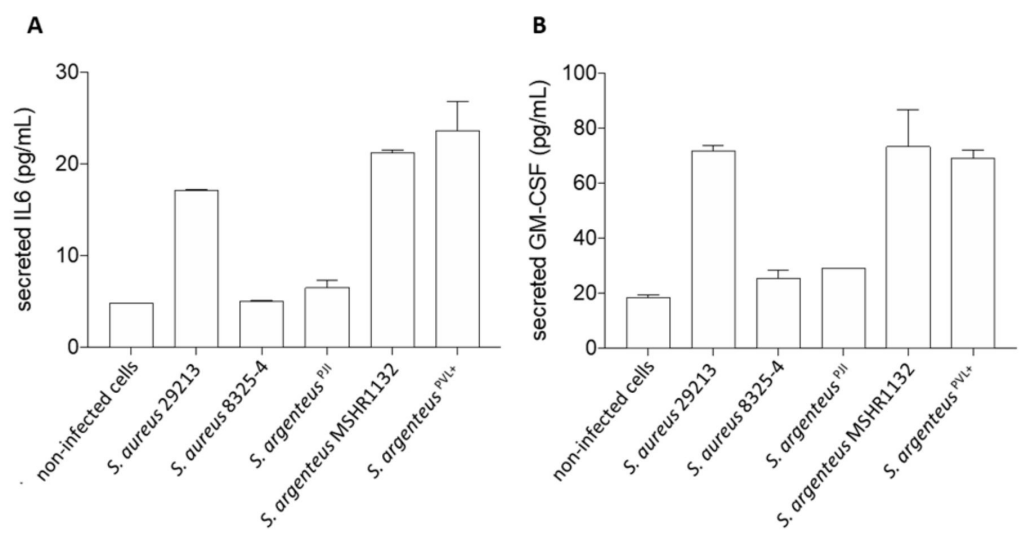

Figure 5. Cellular inflammation induced by the S. argenteus $^{\mathrm{PJI}}$ strain. The secretion of interleukin-6 (A) and GM-CSF (B) by MG63 cells was measured using ELISA assay. S. argenteus ${ }^{\mathrm{PJI}}$ displays a low level of induced inflammation compared to the other invasive strains (S. aureus 29213, S. argenteus MSHR1132 and S. argenteus ${ }^{\mathrm{PVL}+}$ ).

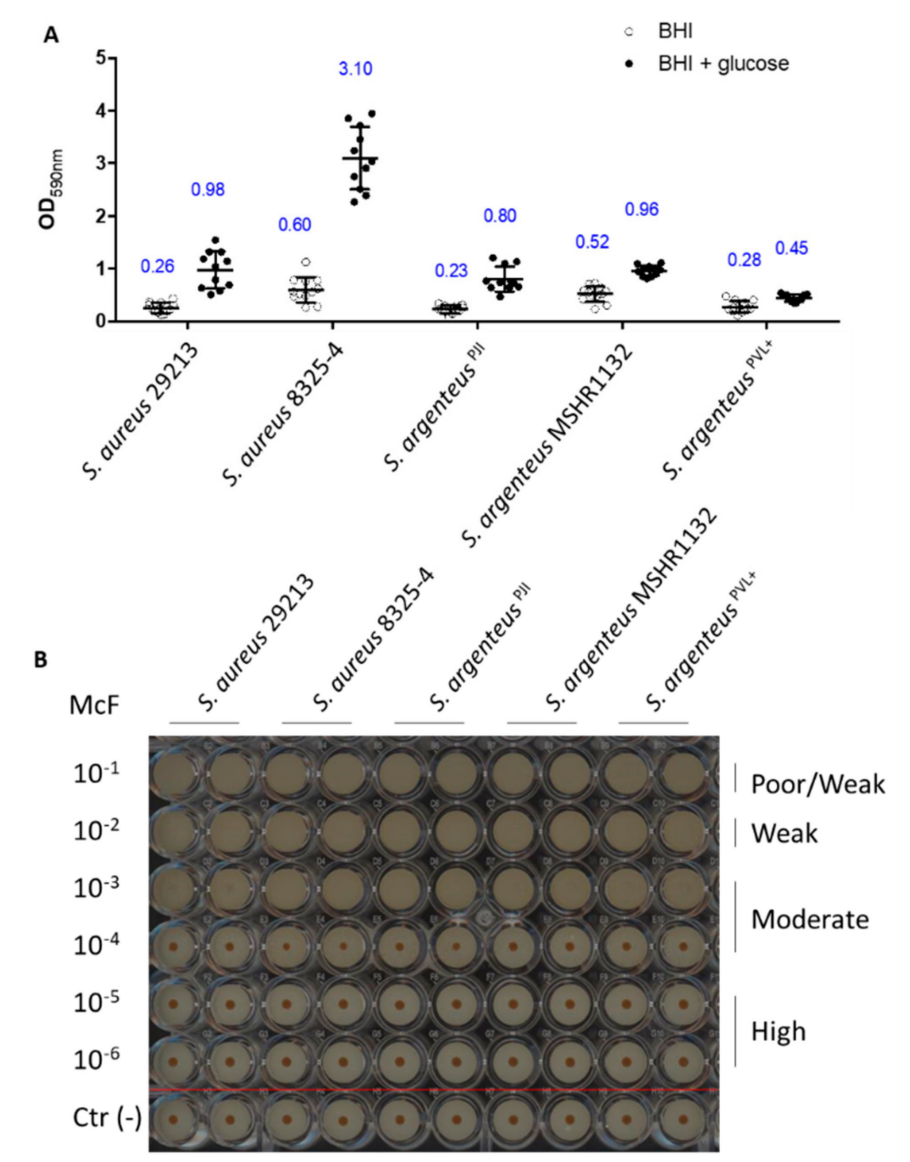

Figure 6. Biofilm formation capacities of the strains. (A) The biofilm formation after $24 \mathrm{~h}$ has been measured using OD measurement at $590 \mathrm{~nm}$ of the crystal violet staining (blue numbers are the $\mathrm{OD}_{590}$ values). (B) The early production of biofilm was assessed using the BioFilm Ring Test ${ }^{\circledR}$ technology and classified based on the previously published procedure [26]. The image is representative of three independent experiments. Negative controls were performed for each condition (below the red line). 
The two other S. argenteus tested (MSHR1132 and S. argenteus $^{P V L+}$ ) also showed an ability to form biofilm similar to the one of the S. aureus strains (Figure 6, $\mathrm{OD}_{590}=0.52$ and 0.28 for MSHR1132 and S. argenteus $^{\mathrm{PVL}+}$ ).

In the presence of glucose, S. argenteus isolates did not respond to a glucose stimulation as much as $S$. aureus strains, especially when compared to $S$. aureus $8325-4$ (Figure $6 \mathrm{~A}, \mathrm{OD}_{590}=0.8$ vs. 0.98 and 3.1 for S. argenteus vs. S. aureus 29213 and S. aureus $8325-4 ; \mathrm{OD}_{590}=0.96$ and 0.45 for MSHR1132 and S. argenteus $^{\mathrm{PVL}+}$ ).

Altogether, these results confirm another asset to the persistence ability of S. argenteus ${ }^{\mathrm{PJI}}$, with a capacity to organize as a biofilm and protect itself from extracellular antimicrobials and the host immune system.

\subsection{Genomic Analysis of Staphylococcus argenteus ${ }^{P I I}$}

As stated above, the genome of $S$. argenteus ${ }^{\mathrm{PJI}}$ was sequenced during a distinct research project in the lab and we took advantage of this to seek for clues on its abilities to persist inside cells and to form biofilm. Anticipated from its original description as being a MSSA, we did not find a SCCmec cassette in S. argenteus ${ }^{\mathrm{PII}}$ genome. However, we found a cassette in the two other S. argenteus strains, even if $S$. argenteus ${ }^{\mathrm{PVL}+}$ is a methicillin-susceptible strain. In S. argenteus ${ }^{\mathrm{PJI}}$, we found genes associated to antibiotic resistances for $\beta$-lactams, erythromycin and fosfomycin. As expected from the $\beta 1$ integrin dependence of its internalization process, both $f n b$ genes were present in the genome of $S$. argenteus ${ }^{\mathrm{PII}}$. Regarding virulence, we did not find the LukS-PV and LukF-PV subunits of the PVL toxin, nor any $\mathrm{PSM} \alpha$ homologs in S. argenteus ${ }^{\mathrm{PJI}}$. However, the PVL subunits were retrieved in the S. aureus $^{P V L+}$ strain. The presence of the ica operon (icaA, C and D), $g b a \mathrm{~A}$ and $-\mathrm{B}, b b p$ and the $p s m \beta$ genes in all the $S$. argenteus was consistent with their ability to form biofilm.

One intriguing element was found in S. argenteus $^{\mathrm{PII}}$ genome; it was located in the region where the SCCmec cassette is present in the two other S. argenteus. In this region, S. argenteus MSHR1132 and S. argenteus ${ }^{\mathrm{PVL}+}$ possess a CRISPR element which is absent in S. argenteus ${ }^{\mathrm{PJI}}$. Instead in S. argenteus ${ }^{\mathrm{PJI}}$, we identified a functional region identified by BLAST as a copper-translocating P-type ATPase. This strain also harbored what seemed to be a putative plasmid bearing an operon that encodes proteins involved in copper and heavy metals transport or detoxification (Figure 7).

A similar plasmid (pvSw4, NCBI access number: NC_020266.1) was identified in S. warneri [28], which covers $73 \%$ and shares $99.48 \%$ of similarity with this one.

Finally, we also identified that the phage $\phi \mathrm{Sa}$, inserted in the genome of the two other S. argenteus in the $\beta$-hemolysin region, is not present in S. argenteus ${ }^{\mathrm{PJI}}$, which results in an intact $\beta$-hemolysin gene. 


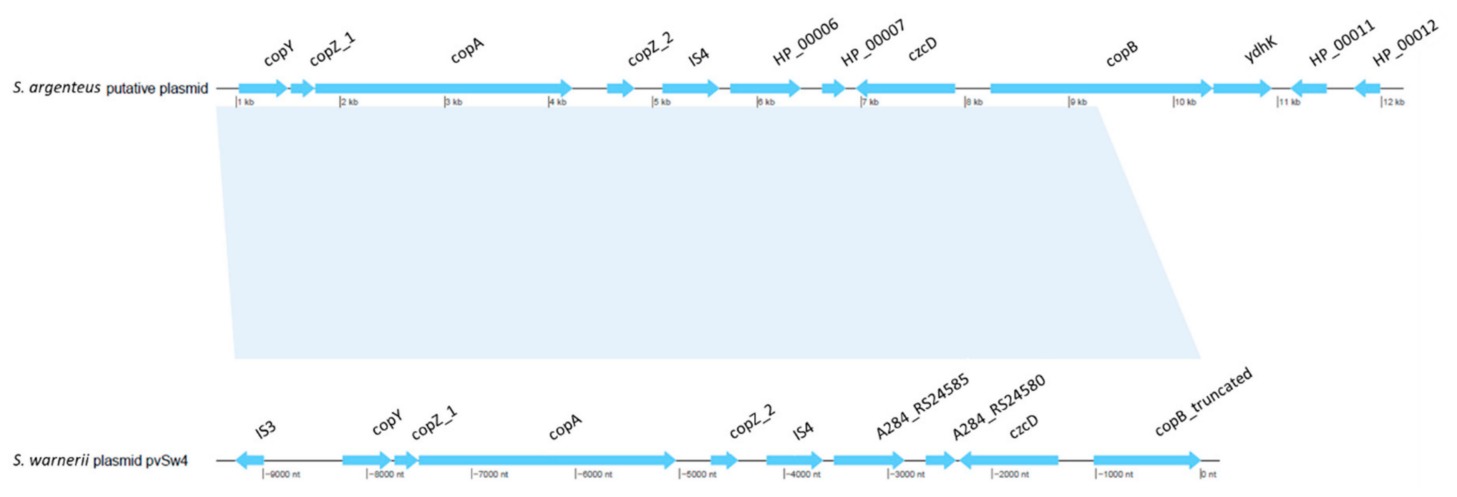

\begin{tabular}{|c|c|}
\hline name & product \\
\hline copY & Transcriptional repressor CopY \\
\hline copZ_1 & Copper chaperone CopZ \\
\hline copA & Copper-exporting P-type ATPase \\
\hline copZ_2 & Copper chaperone CopZ \\
\hline IS4 & IS4 family transposase ISBce8 \\
\hline HP_00006 & hypothetical protein \\
\hline HP_00007 & hypothetical protein \\
\hline czcD & Cadmium, cobalt and zinc/H(+)-K(+) antiporter \\
\hline copB & Copper-exporting P-type ATPase B \\
\hline ydhK & putative protein YdhK \\
\hline HP_00011 & hypothetical protein \\
\hline HP_00012 & hypothetical protein \\
\hline
\end{tabular}

Figure 7. Identification of a putative plasmid in the S. argenteus ${ }^{\mathrm{PJI}}$. A putative plasmid (top) was identified during the genome analysis, similar to the plasmid pvSw4 (bottom, NCBI access number: NC_020266.1) found in S. warneri [28]). The latter covers $73 \%$ and shares $99.48 \%$ of similarity with the one we identified in S. argenteus PJI. These plasmids encode for proteins mainly involved in copper or heavy metals transport (table).

\section{Discussion}

We extensively describe here the first case of an insidious and chronic PJI caused by S. argenteus which happened after three episodes of MSSA infections and could finally be cured by a two-stage exchange strategy with prolonged antimicrobial therapy. Although all the staphylococcal strains identified during the first three episodes of infection were not available, we supposed that they might have been the same $S$. argenteus initially misidentified as $S$. aureus. This statement is reinforced by investigations of bacterial factors associated with BJI chronicity, including bone cell invasion and biofilm formation, revealing that this clinical isolate was well-equipped to be responsible for chronic infections.

The $S$. argenteus ${ }^{\mathrm{PJI}}$ strain was able to invade cells in an efficient way, even if not as efficiently as the two other S. argenteus isolates or the invasive S. aureus 29213. Interestingly, although its intracellular inoculum was high after three days of infection, it induced only a low-grade inflammation. This ability to keep a low inflammatory profile seemed specific to this strain, as the two other $S$. argenteus strains induced a high inflammation. S. argenteus ${ }^{\mathrm{PJI}}$ was also able to form biofilm in vitro and was classified as a moderate producer according to the procedure described in [26]. Altogether, these results suggest that S. argenteus ${ }^{\mathrm{PJI}}$ harbors phenotypic characteristics associated with chronic BJI, combining non-phagocytic bone cell invasion and biofilm formation, with a very limited induction of the cellular inflammatory response. Moreover, the mechanism of bone cell invasion by S. argenteus likely relies on similar 
mechanisms that $S$. aureus, involving staphylococcal FnBP-like proteins and $\alpha 5 \beta 1$ cellular integrin, as suggested using blocking anti- $\beta 1$ integrin antibodies.

Finally, the genome analysis revealed important information. First, we identified that this strain is equipped with proteins allowing to deal with copper or heavy metals detoxification or intake: a copper-translocating P-type ATPase in the genome as well as a putative plasmid encoding for ATPase copper exporter together with the related chaperones (copA, copB, copY and copZ) and proteins involved in the regulation of heavy metals resistance such as $\mathrm{czcD}$ [29]. This extra set of proteins required for copper tolerance could likely represent an effective strategy against the copper killing mechanisms [30-32] used by the host cells to eradicate the bacteria or, at the opposite, could help survive the copper limitation the host could use as another defensive mechanism [33]. Notably, it could play a role similar to the $\operatorname{cop} B$ gene, $m c o$ operon and its repressor $c s o R$ identified on a mobile genetic element in methicillin-resistant $S$. aureus that increase survival in human blood and resistance to killing by host immune cells [34]. We also identified the presence of the $\beta$-hemolysin gene that has been involved in the suppression of the IL-8 production and in the inhibition of the neutrophil migration [35] adding to its low inflammatory potential.

In summary, both genetic features could participate to its good ability to survive in the host by resisting the host defenses and limiting the inflammation which would result in the chronicity of the infection.

Altogether, our study suggests that $S$. argenteus can be implicated in chronic PJI, as harboring phenotypic characteristics associated with immune response and antimicrobial escapement observed in persisting BJI. Based on our findings, we could make the hypothesis that an enhanced resistance to copper together with a propensity to keep a low profile in terms of inflammation induction could have bought this $S$. argenteus ${ }^{\mathrm{PJI}}$ enough time inside the patient to invade cells or form biofilm. Once embedded in a biofilm or inside the intracellular compartment, the bacteria would have been better protected from the extracellular antibiotics and less easily accessible to intracellular antibiotics. This would have resulted in its persistence without clinical signs for the first six months of the episode and might explain the chronicity and the length of the anti-microbials treatments needed, as well as the requirement for prothesis ablation and reimplantation to get rid of this infection. Obviously, more work will be needed to test this hypothesis and identify the factors involved notably in the weak cellular response induced by this invasive S. argenteus PJI strain.

\section{Materials and Methods}

\subsection{Cell Line and Bacteria Strains}

The osteoblastic cell line MG63 (LGC standards, Molsheim, France) was used to explore adhesion, internalization, persistence of staphylococcal strains and the cellular response and cytotoxicity induced by the infection. In addition to $S$. argenteus ${ }^{\mathrm{PJI}}$, the strain of interest isolated during the relapse of the PJI, we used 2 other strains of $S$. argenteus, including the methicillin-resistant strain type MSHR1132 [1,36] and a clinical PVL-positive methicillin-susceptible strain $\left(\right.$ S. argenteus $\left.^{\mathrm{PVL}+}\right)$ [20]. The S. aureus type strain ATCC 29213 and NCTC 8325-4, routinely used in our lab, were used as references.

\subsection{Cell Culture Conditions}

Cells were cultured in $75 \mathrm{~cm}^{2}$ flasks (T75, BD Falcon, Le Pont de Claix, France at $36{ }^{\circ} \mathrm{C}$ under $5 \%$ $\mathrm{CO}_{2}$, in a complete culture medium composed of DMEM (Dulbecco's Modified Eagle Medium, 31885, containing D-glucose, L-glutamine, pyruvate) supplemented with $10 \%$ fetal calf serum (FCS, 10270106), penicillin $(100 \mu \mathrm{g} / \mathrm{mL})$ and streptomycin $(100 \mu \mathrm{g} / \mathrm{mL})$, all from Gibco (Paisley, UK). Cells were passaged once a week and used until passage 25 at most. 


\subsection{Infection and Gentamicin Protection Assay}

MG63 cells were seeded at 100,000 cells per well in a 24-well plate and incubated for $24 \mathrm{~h}$, before being infected at a MOI of 100:1 with the different strains. After $2 \mathrm{~h}$ of contact, cells were washed twice and incubated for $1 \mathrm{~h}$ with a high concentration of gentamicin $(200 \mu \mathrm{g} / \mathrm{mL})$ in order to eliminate adherent and non-adherent extracellular bacteria. For the persistence assessment, the media was replaced with the same fresh media supplemented with gentamicin $(40 \mu \mathrm{g} / \mathrm{mL})$ (to prevent possible reinfection of new cells after lysis of infected cells liberating intracellular bacteria), and changed every $48 \mathrm{~h}$ except for the cytokine secretion measurement (see below). Cells were lysed by osmotic shock in water after $2 \mathrm{~h}, 3 \mathrm{~h}, 24 \mathrm{~h}, 72 \mathrm{~h}$ and 7 days, and lysates were plated on TSA agar plates using the Easy Spiral ${ }^{\circledR}$ automaton (Interscience, Saint-Nom-la-Bretèche, France). CFU were counted after $18 \mathrm{~h}$ of incubation at $36^{\circ} \mathrm{C}$. Bacterial adhesion was calculated by subtracting the CFU counted at $3 \mathrm{~h}$ to those counted after $2 \mathrm{~h}$.

\subsection{Cell Death Assessment}

The cell death was evaluated by the quantification of the lactate dehydrogenase (LDH) released in the extracellular medium using the Cytotoxicity Detection kit (Roche, Basel, Switzerland, 11644793001) following the manufacturer recommendations.

\subsection{Cytokines Secretion by Infected Osteoblasts}

GM-CSF and Il-6 secretions were quantified in the cell culture supernatant after $72 \mathrm{~h}$ of infection without media change, using the human GM-CSF and IL-6 DuoSet ELISA kit from R\&D Systems (refs. DY215 and DY206) and according to the manufacturer's recommendations.

\subsection{Biofilm Formation}

Early biofilm formation capacity was tested using the BioFilm Ring Test ${ }^{\circledR}$ technology (BioFilm Control, Saint Beauzire, France), a magnetic bead immobilization assay which allows the classification of strains according to the previously published procedure [26].

Mature biofilm production was quantified using crystal violet staining associated with the steam-based washing method (BiofilmCare) as described previously [37].

\subsection{Whole Genome Sequencing}

Genomic DNA was extracted from each isolate using a QIAcube extraction kit (Qiagen, Hilden, Germany) and sequencing libraries were prepared from $1 \mathrm{ng}$ of DNA using the Nextera XT DNA preparation kit (Illumina). Whole-genome sequencing was done with an Illumina MiSeq (Illumina, San Diego, CA, USA) to generate 300-bp paired-end reads that were de novo assembled using Unicycler v 0.4.5 pipeline [38] with default parameters (including read's correction and trimming steps before the assembly, scaffolding, removing overlap and bridging steps). A quality assessment of the assemblies was performed with QUAST v 4.6.3 [39].

The genome of the chronic $S$. argenteus clinical isolate was compared with those of the S. argenteus type strain MSHR1132 [1,36] (GenBank acc. num. FR821777,2), a previously described PVL+ strain [20] and the S. aureus strain NCTC8325 (GenBank acc. num. NC_0077951).

The presence or absence of $38 \mathrm{~S}$. aureus genes known to be involved in internalization, biofilm production, persistence and virulence was investigated using ABRicate v 0.8.1 [40] with default parameters (Table 2). 
Table 2. Presence (+) or absence (-) of genes involved in S. aureus virulence, in the S. argenteus strains used.

\begin{tabular}{|c|c|c|c|}
\hline & S. argenteus ${ }^{\mathrm{PJI}}$ & S. argenteus MSHR1132 & S. argenteus $\mathrm{PVL}+$ \\
\hline \multicolumn{4}{|c|}{ PSM genes } \\
\hline PSM_alpha1 & + & + & + \\
\hline PSM_alpha2 & + & + & + \\
\hline PSM_alpha3 & + & + & + \\
\hline PSM_alpha4 & + & + & - \\
\hline PSM_beta1 & + & + & + \\
\hline \multicolumn{4}{|c|}{ Biofilm formation } \\
\hline ebpS & + & + & + \\
\hline icaA & + & + & + \\
\hline icaB & + & + & + \\
\hline icaC & + & + & + \\
\hline fib & + & + & + \\
\hline eno & + & + & + \\
\hline bbp & + & + & + \\
\hline clfA & + & + & - \\
\hline clfB & + & + & - \\
\hline \multicolumn{4}{|c|}{ Internalisation } \\
\hline fnbpA & + & + & + \\
\hline fnbpB & + & + & + \\
\hline atl & + & + & + \\
\hline clfA & + & + & + \\
\hline sdrD & + & + & - \\
\hline tet38 & + & + & + \\
\hline sraP & + & + & - \\
\hline eap & + & + & + \\
\hline gapC & + & + & + \\
\hline \multicolumn{4}{|c|}{ Persistence } \\
\hline $\operatorname{sdh} A$ & + & + & + \\
\hline sdhB & + & + & + \\
\hline ureG & + & + & + \\
\hline $\mathrm{mnhG}$ & + & + & + \\
\hline $\mathrm{fbaA}$ & + & + & + \\
\hline $\mathrm{ctaB}$ & + & + & + \\
\hline mazF & + & + & + \\
\hline $\operatorname{glpX}$ & + & + & + \\
\hline $\operatorname{clpX}$ & + & + & + \\
\hline parE & + & + & + \\
\hline \multicolumn{4}{|c|}{ Leukocidins } \\
\hline hglA & + & + & + \\
\hline hglB & + & + & + \\
\hline hglC & + & + & + \\
\hline
\end{tabular}


Table 2. Cont.

\begin{tabular}{ccccc}
\hline & S. argenteus $^{\mathrm{PJI}}$ & \multicolumn{2}{c}{ S. argenteus MSHR1132 $^{\text {S. }}$} & argenteus $^{\mathrm{PVL}+}$ \\
\hline & & Virulence & \\
\hline $\operatorname{sigB}$ & + & & + & + \\
\hline agrA & + & & + & + \\
\hline
\end{tabular}

The resistome of all S. argenteus genomes was assessed with SRST2 using the ResFinder [41] database. The characterization of SCCmec and CRISPR elements were performed, respectively, with SCCmecFinder [42] and CRISPRcasFinder [43]. The presence of the small phenol soluble modulins alpha 1-4 (PSM $\alpha 1-4)$ was assessed by tblastn using the protein sequences of MSHR1132 PSM $\alpha$ as query. The genome annotation was made with Prokka v 1.13.3 [44], and Mauve progressive [45] alignments were used to check the location of the annotated leukocidins. The differential presence of genes between $S$. argenteus and S. aureus and between the chronic $S$. argenteus and the other S. argenteus was assessed with Roary v3.11.2 [46]. The presence of phage was tested using Phaster [47].

\subsection{Study Protocol and Statistical Analysis}

For phenotype experiments, three independent experiments were performed for each read-out in technical triplicate ( 3 wells for each conditions) except for cytokine secretion measurements that were only performed on supernatants from 2 independent experiments. For internalization and persistence assays, results are presented as the number of intracellular staphylococci for 100,000 osteoblastic cells. Due to the observational nature of this study, we chose to not perform statistical analysis, except for the results in Figure 3.

Author Contributions: Conceptualization, A.D. and F.L.; methodology, A.D. and V.D.-T; software, M.B and P.M.-S.; validation, A.D., J.J., F.V. and F.L.; formal analysis, A.D.; investigation, A.D., V.D.-T. and J.T.; resources, F.L.; data curation, A.D., V.D.-T.; M.B., J.T., P.M.-S., J.J. and F.V.; writing-original draft preparation, A.D.; writing-review and editing, A.D., V.D.-T; M.B., J.T., P.M.-S., J.J., F.V. and F.L.; visualization, M.B. and P.M.-S.; supervision, A.D. and F.L.; project administration, J.J.; and funding acquisition, F.L. All authors have read and agreed to the published version of the manuscript.

Funding: This research received no external funding.

Acknowledgments: We acknowledge the members of National Reference Center for Staphylococci for their technical help.

Conflicts of Interest: The authors declare no conflict of interest.

\section{Abbreviations}

$\begin{array}{ll}\text { BJI } & \text { Bone and Joint Infection } \\ \text { CFU } & \text { Colony Forming Unit } \\ \text { FnBP } & \text { Fibronectin Binding Protein } \\ \text { GM-CSF } & \text { Granulocyte Macrophage Colony Stimulating Factor } \\ \text { LDH } & \text { Lactate Deshydrogenase } \\ \text { MRSA } & \text { Meticillin Resistant Staphylococcus aureus } \\ \text { MSCRAMM } & \text { microbial surface components recognizing adhesive matrix molecule } \\ \text { MSSA } & \text { Meticillin Susceptible Staphylococcus aureus } \\ \text { NPPC } & \text { Non Professional Phagocytic Cell } \\ \text { PJI } & \text { Prosthetic Joint Infection } \\ \text { PSM } & \text { Phenol Soluble Modulin } \\ \text { PVL } & \text { Panton Valentine Leukocidin } \\ \text { SCV } & \text { Small Colony Variant }\end{array}$




\section{References}

1. Holt, D.C.; Holden, M.T.G.; Tong, S.Y.C.; Castillo-Ramirez, S.; Clarke, L.; Quail, M.A.; Currie, B.J.; Parkhill, J.; Bentley, S.D.; Feil, E.J.; et al. A Very Early-Branching Staphylococcus aureus Lineage Lacking the Carotenoid Pigment Staphyloxanthin. Genome Biol. Evol. 2011, 3, 881-895. [CrossRef] [PubMed]

2. Moradigaravand, D.; Jamrozy, D.; Mostowy, R.; Anderson, A.; Nickerson, E.K.; Thaipadungpanit, J.; Wuthiekanun, V.; Limmathurotsakul, D.; Tandhavanant, S.; Wikraiphat, C.; et al. Evolution of the Staphylococcus argenteus ST2250 Clone in Northeastern Thailand Is Linked with the Acquisition of Livestock-Associated Staphylococcal Genes. mBio 2017, 8. [CrossRef] [PubMed]

3. Ritchie, S.R.; Thomas, M.G.; Rainey, P.B. The genetic structure of Staphylococcus aureus populations from the Southwest Pacific. PLoS ONE 2014, 9, e100300. [CrossRef] [PubMed]

4. Argudín, M.A.; Dodémont, M.; Vandendriessche, S.; Rottiers, S.; Tribes, C.; Roisin, S.; de Mendonça, R.; Nonhoff, C.; Deplano, A.; Denis, O. Low occurrence of the new species Staphylococcus argenteus in a Staphylococcus aureus collection of human isolates from Belgium. Eur. J. Clin. Microbiol. Infect Dis. 2016, 35, 1017-1022. [CrossRef] [PubMed]

5. Rigaill, J.; Grattard, F.; Grange, S.; Forest, F.; Haddad, E.; Carricajo, A.; Tristan, A.; Laurent, F.; Botelho-Nevers, E.; Verhoeven, P.O. Community-Acquired Staphylococcus argenteus Sequence Type 2250 Bone and Joint Infection, France, 2017. Emerg. Infect Dis. 2018, 24, 1958-1961. [CrossRef] [PubMed]

6. Foster, T.J.; Geoghegan, J.A.; Ganesh, V.K.; Höök, M. Adhesion, invasion and evasion: The many functions of the surface proteins of Staphylococcus aureus. Nat. Rev. Microbiol. 2014, 12, 49-62. [CrossRef]

7. Löffler, B.; Tuchscherr, L.; Niemann, S.; Peters, G. Staphylococcus aureus persistence in non-professional phagocytes. Int. J. Med. Microbiol. 2014, 304, 170-176. [CrossRef]

8. Ricciardi, B.F.; Muthukrishnan, G.; Masters, E.; Ninomiya, M.; Lee, C.C.; Schwarz, E.M. Staphylococcus aureus Evasion of Host Immunity in the Setting of Prosthetic Joint Infection: Biofilm and Beyond. Curr. Rev. Musculoskelet. Med. 2018. [CrossRef]

9. Abel, J.; Goldmann, O.; Ziegler, C.; Höltje, C.; Smeltzer, M.S.; Cheung, A.L.; Bruhn, D.; Rohde, M.; Medina, E. Staphylococcus aureus Evades the Extracellular Antimicrobial Activity of Mast Cells by Promoting Its Own Uptake. JIN 2011, 3, 495-507. [CrossRef]

10. Moormeier, D.E.; Bayles, K.W. Staphylococcus aureus biofilm: A complex developmental organism. Mol. Microbiol. 2017. [CrossRef]

11. Tuchscherr, L.; Medina, E.; Hussain, M.; Völker, W.; Heitmann, V.; Niemann, S.; Holzinger, D.; Roth, J.; Proctor, R.A.; Becker, K.; et al. Staphylococcus aureus phenotype switching: An effective bacterial strategy to escape host immune response and establish a chronic infection. EMBO Mol. Med. 2011, 3, 129-141. [CrossRef] [PubMed]

12. Tran Van Nhieu, G.; Isberg, R.R. Bacterial internalization mediated by beta 1 chain integrins is determined by ligand affinity and receptor density. EMBO J. 1993, 12, 1887-1895. [CrossRef] [PubMed]

13. Sinha, B.; François, P.P.; Nüße, O.; Foti, M.; Hartford, O.M.; Vaudaux, P.; Foster, T.J.; Lew, D.P.; Herrmann, M.; Krause, K.-H. Fibronectin-binding protein acts as Staphylococcus aureus invasin via fibronectin bridging to integrin $\alpha 5 \beta 1$. Cell. Microbiol. 1999, 1, 101-117. [CrossRef] [PubMed]

14. Grundmeier, M.; Hussain, M.; Becker, P.; Heilmann, C.; Peters, G.; Sinha, B. Truncation of Fibronectin-Binding Proteins in Staphylococcus aureus Strain Newman Leads to Deficient Adherence and Host Cell Invasion Due to Loss of the Cell Wall Anchor Function. Infect Immun. 2004, 72, 7155-7163. [CrossRef] [PubMed]

15. Suci, P.A.; Mittelman, M.W.; Yu, F.P.; Geesey, G.G. Investigation of ciprofloxacin penetration into Pseudomonas aeruginosa biofilms. Antimicrob. Agents Chemother. 1994, 38, 2125-2133. [CrossRef] [PubMed]

16. Mah, T.-F.; Pitts, B.; Pellock, B.; Walker, G.C.; Stewart, P.S.; O'Toole, G.A. A genetic basis for Pseudomonas aeruginosa biofilm antibiotic resistance. Nature 2003, 426, 306-310. [CrossRef] [PubMed]

17. Evans, D.J.; Brown, M.R.; Allison, D.G.; Gilbert, P. Susceptibility of bacterial biofilms to tobramycin: Role of specific growth rate and phase in the division cycle. J. Antimicrob. Chemother. 1990, 25, 585-591. [CrossRef]

18. Zhang, L.; Mah, T.-F. Involvement of a Novel Efflux System in Biofilm-Specific Resistance to Antibiotics. J. Bacteriol. 2008, 190, 4447-4452. [CrossRef]

19. Thurlow, L.R.; Hanke, M.L.; Fritz, T.; Angle, A.; Aldrich, A.; Williams, S.H.; Engebretsen, I.L.; Bayles, K.W.; Horswill, A.R.; Kielian, T. Staphylococcus aureus biofilms prevent macrophage phagocytosis and attenuate inflammation in vivo. J. Immunol. 2011, 186, 6585-6596. [CrossRef] 
20. Dupieux, C.; Blondé, R.; Bouchiat, C.; Meugnier, H.; Bes, M.; Laurent, S.; Vandenesch, F.; Laurent, F.; Tristan, A. Community-acquired infections due to Staphylococcus argenteus lineage isolates harbouring the Panton-Valentine leucocidin, France, 2014. Eurosurveillance 2015, 20, 21154. [CrossRef]

21. Wakabayashi, Y.; Umeda, K.; Yonogi, S.; Nakamura, H.; Yamamoto, K.; Kumeda, Y.; Kawatsu, K. Staphylococcal food poisoning caused by Staphylococcus argenteus harboring staphylococcal enterotoxin genes. Int. J. Food Microbiol. 2018, 265, 23-29. [CrossRef] [PubMed]

22. Aung, M.S.; San, T.; Aye, M.M.; Mya, S.; Maw, W.W.; Zan, K.N.; Htut, W.H.W.; Kawaguchiya, M.; Urushibara, N.; Kobayashi, N. Prevalence and Genetic Characteristics of Staphylococcus aureus and Staphylococcus argenteus Isolates Harboring Panton-Valentine Leukocidin, Enterotoxins, and TSST-1 Genes from Food Handlers in Myanmar. Toxins 2017, 9, 241. [CrossRef]

23. Jiang, B.; You, B.; Tan, L.; Yu, S.; Li, H.; Bai, G.; Li, S.; Rao, X.; Xie, Z.; Shi, X.; et al. Clinical Staphylococcus argenteus Develops to Small Colony Variants to Promote Persistent Infection. Front. Microbiol. $2018,9$. [CrossRef] [PubMed]

24. Monecke, S.; Jatzwauk, L.; Weber, S.; Slickers, P.; Ehricht, R. DNA microarray-based genotyping of methicillin-resistant Staphylococcus aureus strains from Eastern Saxony. Clin. Microbiol. Infect. 2008, 14, 534-545. [CrossRef] [PubMed]

25. Monecke, S.; Slickers, P.; Ehricht, R. Assignment of Staphylococcus aureus isolates to clonal complexes based on microarray analysis and pattern recognition. FEMS Immunol. Med. Microbiol. 2008, 53, 237-251. [CrossRef]

26. Di Domenico, E.G.; Toma, L.; Provot, C.; Ascenzioni, F.; Sperduti, I.; Prignano, G.; Gallo, M.T.; Pimpinelli, F.; Bordignon, V.; Bernardi, T.; et al. Development of an in vitro Assay, Based on the BioFilm Ring Test ${ }^{\circledR}$, for Rapid Profiling of Biofilm-Growing Bacteria. Front. Microbiol. 2016, 7. [CrossRef]

27. Waldrop, R.; McLaren, A.; Calara, F.; McLemore, R. Biofilm Growth Has a Threshold Response to Glucose in Vitro. Clin. Orthop. Relat. Res. 2014, 472, 3305-3310. [CrossRef]

28. Cheng, V.W.T.; Zhang, G.; Oyedotun, K.S.; Ridgway, D.; Ellison, M.J.; Weiner, J.H. Complete Genome of the Solvent-Tolerant Staphylococcus warneri Strain SG1. Genome Announc. 2013, 1. [CrossRef]

29. Anton, A.; Große, C.; Reißmann, J.; Pribyl, T.; Nies, D.H. CzcD Is a Heavy Metal Ion Transporter Involved in Regulation of Heavy Metal Resistance in Ralstonia sp. Strain CH34. J. Bacteriol. 1999, 181, 6876-6881. [CrossRef]

30. Ding, C.; Festa, R.A.; Chen, Y.-L.; Espart, A.; Palacios, Ò.; Espín, J.; Capdevila, M.; Atrian, S.; Heitman, J.; Thiele, D.J. Cryptococcus neoformans copper detoxification machinery is critical for fungal virulence. Cell Host. Microbe 2013, 13, 265-276. [CrossRef]

31. Wiemann, P.; Perevitsky, A.; Lim, F.Y.; Shadkchan, Y.; Knox, B.P.; Landero Figueora, J.A.; Choera, T.; Niu, M.; Steinberger, A.J.; Wüthrich, M.; et al. Host copper-mediated oxidative antimicrobial offense is countered by Aspergillus fumigatus copper export machinery and reactive oxygen intermediate defense. Cell Rep. 2017, 19, 1008-1021. [CrossRef] [PubMed]

32. Wolschendorf, F.; Ackart, D.; Shrestha, T.B.; Hascall-Dove, L.; Nolan, S.; Lamichhane, G.; Wang, Y.; Bossmann, S.H.; Basaraba, R.J.; Niederweis, M. Copper resistance is essential for virulence of Mycobacterium tuberculosis. Proc. Natl. Acad. Sci. USA 2011, 108, 1621-1626. [CrossRef] [PubMed]

33. Li, C.X.; Gleason, J.E.; Zhang, S.X.; Bruno, V.M.; Cormack, B.P.; Culotta, V.C. Candida albicans adapts to host copper during infection by swapping metal cofactors for superoxide dismutase. Proc. Natl. Acad. Sci. USA 2015, 112, E5336-E5342. [CrossRef] [PubMed]

34. Zapotoczna, M.; Riboldi, G.P.; Moustafa, A.M.; Dickson, E.; Narechania, A.; Morrissey, J.A.; Planet, P.J.; Holden, M.T.G.; Waldron, K.J.; Geoghegan, J.A. Mobile-Genetic-Element-Encoded Hypertolerance to Copper Protects Staphylococcus aureus from Killing by Host Phagocytes. MBio 2018, 9. [CrossRef]

35. Tajima, A.; Iwase, T.; Shinji, H.; Seki, K.; Mizunoe, Y. Inhibition of endothelial interleukin-8 production and neutrophil transmigration by Staphylococcus aureus beta-hemolysin. Infect. Immun. 2009, 77, 327-334. [CrossRef]

36. Tong, S.Y.C.; Schaumburg, F.; Ellington, M.J.; Corander, J.; Pichon, B.; Leendertz, F.; Bentley, S.D.; Parkhill, J.; Holt, D.C.; Peters, G.; et al. Novel staphylococcal species that form part of a Staphylococcus aureus-related complex: The non-pigmented Staphylococcus argenteus sp. nov. and the non-human primate-associated Staphylococcus schweitzeri sp. nov. Int. J. Syst. Evol. Microbiol. 2015, 65, 15-22. [CrossRef] 
37. Tasse, J.; Cara, A.; Saglio, M.; Villet, R.; Laurent, F. A steam-based method to investigate biofilm. Sci. Rep. 2018, 8, 13040. [CrossRef]

38. Wick, R.R.; Judd, L.M.; Gorrie, C.L.; Holt, K.E. Unicycler: Resolving bacterial genome assemblies from short and long sequencing reads. PLoS Comput. Biol. 2017, 13, e1005595. [CrossRef]

39. Gurevich, A.; Saveliev, V.; Vyahhi, N.; Tesler, G. QUAST: Quality assessment tool for genome assemblies. Bioinformatics 2013, 29, 1072-1075. [CrossRef]

40. Seemann, T. Abricate, Github. Available online: https://github.com/tseemann/abricate (accessed on 28 August 2020).

41. Zankari, E.; Hasman, H.; Cosentino, S.; Vestergaard, M.; Rasmussen, S.; Lund, O.; Aarestrup, F.M.; Larsen, M.V. Identification of acquired antimicrobial resistance genes. J. Antimicrob. Chemother. 2012, 67, 2640-2644. [CrossRef]

42. Kaya, H.; Hasman, H.; Larsen, J.; Stegger, M.; Johannesen, T.B.; Allesøe, R.L.; Lemvigh, C.K.; Aarestrup, F.M.; Lund, O.; Larsen, A.R. SCCmecFinder, a Web-Based Tool for Typing of Staphylococcal Cassette Chromosome mec in Staphylococcus aureus Using Whole-Genome Sequence Data. mSphere 2018, 3. [CrossRef] [PubMed]

43. Couvin, D.; Bernheim, A.; Toffano-Nioche, C.; Touchon, M.; Michalik, J.; Néron, B.; Rocha, E.P.C.; Vergnaud, G.; Gautheret, D.; Pourcel, C. CRISPRCasFinder, an update of CRISRFinder, includes a portable version, enhanced performance and integrates search for Cas proteins. Nucleic Acids Res. 2018, 46, W246-W251. [CrossRef] [PubMed]

44. Seemann, T. Prokka: Rapid prokaryotic genome annotation. Bioinformatics 2014, 30, 2068-2069. [CrossRef] [PubMed]

45. Tritt, A.; Eisen, J.A.; Facciotti, M.T.; Darling, A.E. An Integrated Pipeline for de Novo Assembly of Microbial Genomes. PLoS ONE 2012, 7, e42304. [CrossRef] [PubMed]

46. Page, A.J.; Cummins, C.A.; Hunt, M.; Wong, V.K.; Reuter, S.; Holden, M.T.G.; Fookes, M.; Falush, D.; Keane, J.A.; Parkhill, J. Roary: Rapid large-scale prokaryote pan genome analysis. Bioinformatics 2015, 31, 3691-3693. [CrossRef]

47. Arndt, D.; Grant, J.R.; Marcu, A.; Sajed, T.; Pon, A.; Liang, Y.; Wishart, D.S. PHASTER: A better, faster version of the PHAST phage search tool. Nucleic Acids Res. 2016, 44, W16-W21. [CrossRef]

(C) 2020 by the authors. Licensee MDPI, Basel, Switzerland. This article is an open access article distributed under the terms and conditions of the Creative Commons Attribution (CC BY) license (http://creativecommons.org/licenses/by/4.0/). 